\title{
Impact of Online Learning on Motivation and Achievement
}

\author{
Amira Khattab $^{1}$, David Egleston ${ }^{2}$ \\ Digital14 $^{1}$, Rochester Institute of Technology Dubai ${ }^{2}$ \\ United Arab Emirates
}

\begin{abstract}
There is a question of whether instructional techniques have the same effect across the face-to-face and computer-mediated modalities. This study examined the effects of synchronicity and belongingness on performance in an undergraduate class in a multicultural environment. Fifty-six undergraduate students from two sections of Business Ethics were randomly assigned to a 3 (synchronicity: face-to-face $(F T F$,$) synchronous computer-mediated$ communication (SCMC), or asynchronous computermediated communication $(A C M C))$ by 3 (belongingness: acceptance, mild rejection, control) quasi-experimental design using the constructive controversy technique. The competition was highest in the FTF condition and critical thinking was lowest in the SCMC condition. Mild rejection reduced attitudes toward technology in the ACMC condition but not in the other two boundary conditions. Cultural variables also affected team outcomes. Students high in uncertainty avoidance were more competitive and demonstrated higher levels of epistemic behavior while those high in collectivism reported improved attitudes and cooperation. Implications for theory and practice are discussed.
\end{abstract}

\section{Introduction}

The unprecedented coronavirus pandemic is profoundly affecting the way we learn, forcing brick and mortar institutions to turn to distance learning. With such imposed digital transformation, students from different demographic backgrounds have no choice but to work together in order to achieve a shared goal despite having varying motivations [1].

We designed this study to address the role of synchronicity, belongingness, and culture when evaluating one's motivation and drive pertaining to academic achievement across instructional modalities.

We examined the impact of the delivery mode for course material utilizing the constructive controversy methodology [2], a robust and empirically sound pedagogy, using three different boundary conditions: face-to-face, synchronous, and asynchronous computer-mediated communications (CMC), previously examined by Saltarelli and Roseth [3]. Saltarelli et al.'s findings demonstrate the deleterious effects of asynchronous communication and the positive impact of belongingness interventions on educational outcomes. In addition, Saltarelli's study suggests constructive controversy may stimulate different psychological processes across boundary conditions. This evidence raises important questions about the extent to which constructive controversy functions equivalently across cultures.

\section{Literature Review}

Constructive controversy is a cooperative learning instructional procedure in which individuals argue incompatible perspectives and together seek to reach an agreement by resolving cognitive conflicts in a cooperative manner [4]. Teachers organize students into cooperative groups of four and divide each group into two pairs, assigning the pro position on a topic to one pair and the con position to the other pair, and then direct students through a structured procedure and strategies for completing the tasks or solving problems. Constructive controversy can be used with any subject matter or topic.

A meta-analysis of 40 years of research demonstrates the efficacy of constructive controversy. Contrasted with concurrence seeking, debate, and individualistic learning, constructive controversy results in higher achievement and greater mastery of content, higher level reasoning and metacognitive thought, more accurate understanding of opposing perspectives, more open-mindedness, increased motivation to learn, higher creativity levels and degrees of commitment to solving problems, more positive relationships and appreciation of other positions, and greater self-esteem and liking among participants holding conflicting stances [5]. Recent research shows that the outcomes of constructive controversy deteriorate under asynchronous CMC [3]. However, none of the studies examine the effective 
implementation of constructive controversy in an online setting and in an Arabic context. As such, we have designed the present study to (a) test whether the findings of Saltarelli and Roseth [3] replicate with an Arabic sample and (b) examine the effects of national culture on psychological and relational processes involved in constructive controversy.

Relational processes underpinning constructive controversy - Social interdependence and sociocognitive conflict regulation [6] assert that enhanced motivation, academic achievement, and attitudes are all outcomes of cooperative perceptions and promotive interaction processes. In social interdependence, goals are divided into two categories. One category is positive social interdependence, when individuals share goals through cooperative behavior [4]. The other category of social interdependence is negative, or when one's goal competes with that of the individual they are working with [4].

Sociocognitive conflict regulation is a theory that looks at conflict of knowledge or what is determined to be correct to individuals working together as identified by Darnon et. al [7]. An epistemic resolution is one that occurs as a result of individuals being focused on a task, while a relational resolution is based on the group's ability to engage in social comparison of competencies [3].

Constructive controversy is linked to social interdependence and sociocognitive conflict regulation by compelling individuals to experience a conflict of opinion and perspectives. Conflict emerges and is resolved in either an epistemic or relational manner [3]. Previous studies on conflict behavior of Arabs, who are considered collectivist rather than independent, in FTF and CMC settings demonstrate an avoidance of conflict in FTF settings [8].

Self-determination theory and belongingness - Ryan and Deci [9] contend that classroom practices, which endorse student autonomy, competency, and relatedness, are critical for enhancing intrinsic motivation to learn and for fostering student socioemotional development. Self-determination theorists conceive of relatedness as a basic human need for all individuals and a vital ingredient in human development. In academic settings, relatedness refers to the social-emotional support students need to effectively approach, engage, and persist in learning tasks, to form positive achievement-related beliefs, and to cultivate a sense of belonging in a learning environment. Belonging is a fundamental human need [9] and satisfying this need is linked to enhanced academic achievement, and motivation [10]. Consequently, educational psychologists assert that the integration of affiliative and achievement constructs [11], domains that have traditionally been separate, is valuable in fostering student motivation to learn and promoting a sense of community, belongingness, relatedness, connectedness, and perceived support.

The evidence suggests that satisfying the needs of students may increase the effectiveness of achievement and motivation aspects of constructive controversy. Nevertheless, the evidence solely applies to research conducted in FTF settings.

Wallace [12] in her literature review of over 200 articles about online teaching and learning, notes that students become frustrated by many aspects of online learning, while course instructors are unaware of student frustration. She concludes that available studies present conflicting views on whether connections and positive relationships can be developed in an online environment.

To our knowledge, only one empirical study investigated whether the effects of belongingness on constructive controversy generalize across both online (synchronous and asynchronous) and FTF settings [3]. Saltarelli and Roseth's study examined one hundred seventy-one undergraduates randomly assigned to FTF, synchronous, and asynchronous groups in a quasiexperimental design. The authors found that satisfying belongingness needs buffers but does not neutralize, the adverse effects of asynchronous CMC.

Computer mediated communication: Effects of synchronicity on cooperative learning - Computermediated communication (CMC) allows individuals to interact and develop relationships with one another, although the interactions are completely disembodied or potentially asynchronous [13]. Synchronous communication in a learning environment could involve a live, real time chat room where the instructor interacts with the students and the students interact with each other. Asynchronous communication in a learning environment could involve a message board, where individuals interact with each other on their own time. Previous studies conducted by Saltarelli and Roseth [3] have demonstrated a shift in outcomes based on how groups experience CMC due to synchronicity. They found that competition and relational conflicts increased, and academic achievement and motivation decreased under asynchronous CMC learning conditions.

Al-Harthi [14] examined cultural variations between Arab and American distance learners to 
explore learner preferences and identify the ways they self-regulate and interact through CMC. American students scored higher than Arab students on planning, monitoring, effort, time and environment management, and self-efficacy, all of which are critical constructs for success in an online environment. Literature related to non-Western cultures has demonstrated that individuals from Arab and East Asian cultures are less likely to speak up when engaging in a new environment out of fear of criticism or providing a wrong answer [15].

Effects of culture - Understanding how culture interacts with intra- and socio-psychological constructs, motives, and processes, allow for (a) theory expansion by examining its robustness, (b) testing the universality of Western-centric constructs, (c) the extension of selfconstrual models, and (d) isolating/controlling for some confounding variables.

King [16] defines culture as "a sense of peoplehood and commonality derived from kinship patterns, a shared historical past, common experiences, religious affiliations, language or linguistic commonalities, shared values, attitudes and perceptions, modes of expression and identity" [p.89]. Hofstede [17] surmises that, while every group or category of people carries a set of common mental programs that form its culture, measurement of culture does not imply that everyone in a given society is programmed in the same way. Statements about culture do not describe individual differences or within-country variances; they are all general and relative and represent nations.

Using Hofstede's typology, Sheldon et al. [18] examined the individualism-collectivism dimension of college-age students from the United States and South Korea. The authors found that both groups ranked autonomy, competence, and relatedness at the top of their lists, and that the three needs explained distinctive variance in student well-being.

Based on a sample of 1521 executives in 11 Arab countries, Khattab [19] found that learning and management approaches vary across Hofstede's cultural dimensions. Data showed that individuals belonging to nations with higher values of Power Distance Index and Uncertainty Avoidance Index were generally inclined towards didactic approaches of learning, autocratic models of leadership, lecture or theory-based instruction, and traditional views for physical spaces and classroom tools.

The previous studies support that culture, and its implications on individual reasoning and worldview, influence the way people approach conflict resolution, debates, relationships with others, and communication skills [20].

To date, constructive controversy and the ways it varies across boundary contexts, have not been empirically examined through a cultural lens. Thus far, constructive controversy and culture have been loosely linked and conceptually discussed in business management literature [21].

\section{Proposed study}

In this study, we examine how boundary conditions (i.e., FTF, synchronous $\mathrm{CMC}$, and asynchronous CMC) affect constructive controversy outcomes. Firstly, we investigate how synchronicity impacts academic achievement, and intrapsychological and relational processes. Secondly, we test whether a belongingness intervention impacts constructive controversy. Thirdly, we examine whether findings vary as a function of national culture.

\subsection{Research questions}

Guided by social interdependence theory and based on the study conducted by Saltarelli [3], we aim to address the following five key research questions:

1. Does constructive controversy stimulate different psychological processes under FTF and CMC conditions, resulting in different outcomes?

2. What are the effects of social interdependence on academic achievement, motivation, and relational processes? 


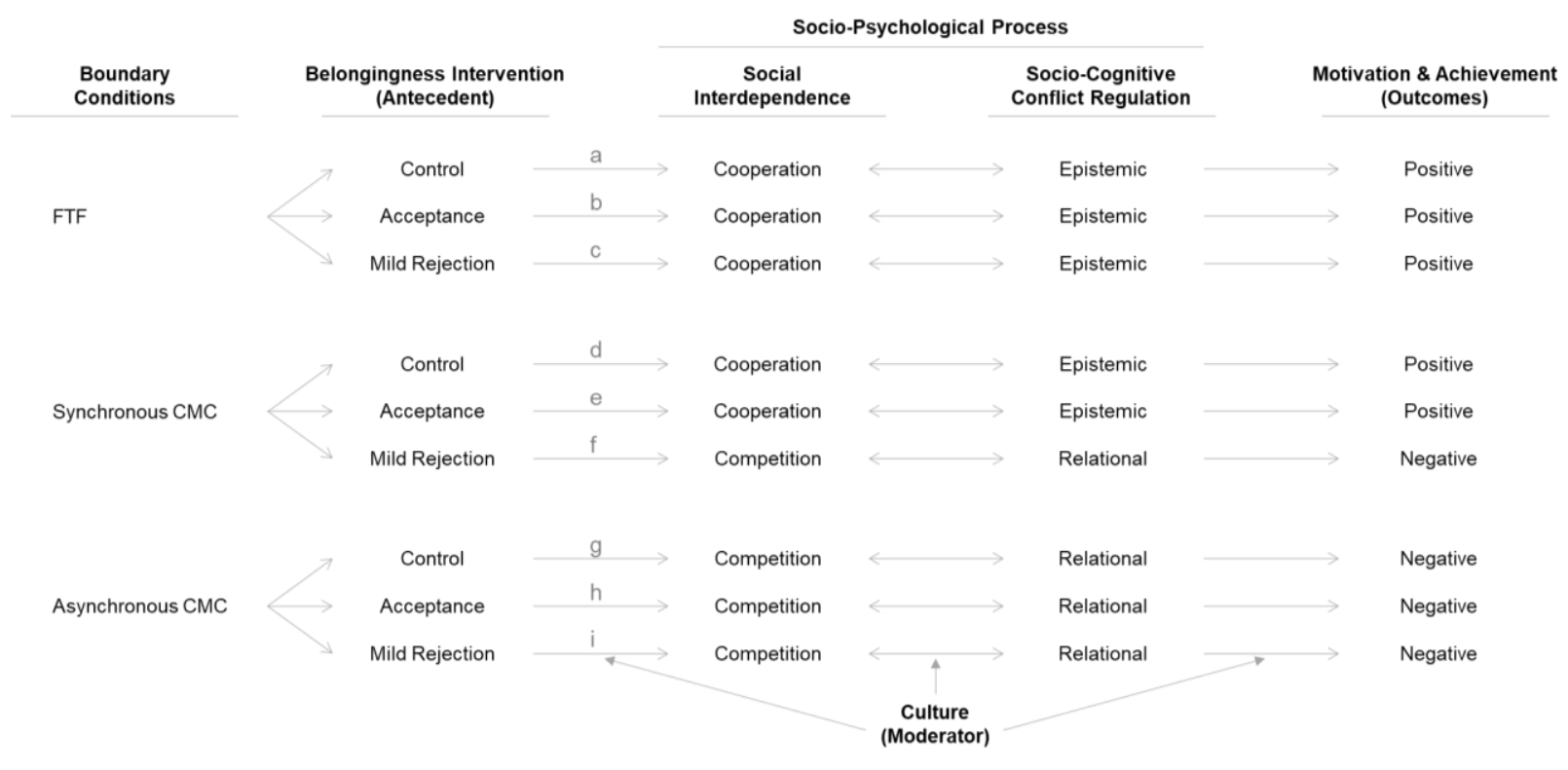

Figure 1. Conceptual model

3. What are the effects of sociocognitive conflict regulation on achievement, motivation, and relational processes?

4. What are the effects of varying levels of belongingness (acceptance and mild rejection) on achievement, motivation, and relational processes? Do these effects vary under FTF and CMC conditions?

5. Do cultural dimensions such as individualismcollectivism (CO) and uncertainty avoidance (UAI) affect constructive controversy outcomes? Do results generalize across boundary conditions?

The Figure 1 illustrates the way FTF, synchronous and asynchronous CMC conditions, a belongingness intervention, and the moderating effects of national culture may impact the relational processes underpinning constructive controversy and, in turn, affect students' motivation and academic achievement. Guided by social interdependence theory and based on the study conducted by Saltarelli and Roseth [3], we predict that asynchronous CMC will promote competition and relational conflict regulation of participants and in turn, there will be a corresponding decrease in motivation and achievement (see Figure 1, part g). Guided by selfdetermination theory, we predict that the acceptance form of the belongingness intervention will buffer any negative effects that may arise from synchronous $\mathrm{CMC}$ and will result in an increase in cooperative and epistemic relational beliefs (see Fig. 1, part e). Additionally, we predict that mild rejection will have a negative effect on cooperation and conflict regulation in both synchronous and asynchronous settings but not in the FTF condition (see Fig. 1, part c). However, due to the moderating effects of culture, we argue that participants with a moderately high $\mathrm{UAI}$ and high CO (more representative of Arab and Asian countries) will not be able to compensate the aversive effects of asynchronous CMC settings. As a result, asynchronous $\mathrm{CMC}$ settings will promote competition and relational conflict regulation and diminish motivation and achievement (see Fig. 1, parts $\mathrm{h}$ and i). We theorize that participant inclination to avoid risks and high propensity to build interpersonal relationships will exacerbate the negative effects of asynchronous CMC.

To enhance the robustness of our findings, we aim to address two limitations of Saltarelli and Roseth's [3] research by: a) ensuring we have a higher ratio of males in our sample; and b) measuring students' perception of technology before and after the experiment to find out whether positive attitude towards technology was a result of the synchronous activity or had an effect on students' performance by acting as an antecedent. 


\section{Methodology}

The study was conducted at a university in Dubai, using undergraduate students enrolled in two sections of the same business ethics course. Participants were randomly assigned to a 3 (synchronicity: FTF, synchronous $\mathrm{CMC}$, asynchronous $\mathrm{CMC}$ ) by 3 (belongingness: acceptance, mild rejection, control) quasi-experimental design. Students were randomly assigned to three synchronicity groups (boundary conditions). Students within each boundary condition were randomly assigned to the three belongingness groups and a pro/con stance with a student with the opposite stance for constructive controversy.

Before the constructive controversy procedure, participants attended a 60-minute session to learn about the study, sign the consent forms, attend a technology training tutorial, receive instructions on the constructive controversy procedure, and complete the Eysenck Personality Questionnaire Brief Version [22]. Several days later, students received a belongingness manipulation message [23] and their randomly assigned partner. Participants also received an email about their group's stance (pro or con) and the pro or con article for the constructive controversy procedure. The articles focused on the ethics of whistleblowing, an appropriate topic for a business ethics course. After the constructive controversy exercise, participants filled out a post-procedure survey to measure social interdependence, sociocognitive conflict regulation, motivation, academic achievement, time spent, and their perceptions of technology. Participants then received a debriefing report.

\subsection{Independent variables}

Synchronicity and belongingness were categorical independent variables, and the two cultural dimensions measuring collectivism and uncertainty avoidance were covariates. The cultural dimension variables were based on scale items ranging from 1 ('Strongly Disagree') to 7 ('Strongly Agree').

The synchronous CMC condition relied primarily on real-time online communication. Communication involved real-time text, audio, or video-based discussion with partners. The asynchronous CMC condition mainly involved text-based communication with partners through emails and an online forum, which facilitated posting messages not occurring at the same time. For FTF, participants utilized class time to communicate.
The belongingness manipulation was administered using a procedure that placed participants in one of three states: control, acceptance, or mild rejection [24].

For the purposes of this study, only two cultural dimensions of Hofstede's six indices were examined: individualism-collectivism index and uncertainty avoidance index. Hofstede [17] created the indices using both theoretical reasoning and statistics. However, these values must be used at the country level, to avoid an ecological fallacy. Therefore, in order to use these cultural dimensions at the individual level, this study used a modified scale that measures Hofstede's cultural dimensions at the individual level. Yoo et. al [25] developed a new scale after collecting hundreds of items from Hofstede's work, as well as from non-Hofstede work that represented the core definitions of his dimensions. Through a series of surveys and multiple samples, the researchers refined the item pool to 26 reliable items that represent Hofstede's cultural dimensions at the individual level. This survey is called the CVSCALE. The CVSCALE [25] was used to measure the individualismcollectivism index (CO) for each participant (5 items; $\alpha=0.66$ ). The higher the value, the more collectivist the individual. The CVSCALE [25] was also used to measure uncertainty avoidance (UAI) for each individual ( 5 items; $\alpha=0.72$ ). The higher the value, the more the individual desires to avoid uncertainty.

\subsection{Dependent variables}

The six dependent variables: social interdependence, sociocognitive conflict regulation, motivation, academic achievement, time, and perceptions of technology. Except for achievement and time, the dependent variables were based on scale items ranging from 1 ('Not at All True' for motivation or 'Strongly Disagree' for all others) to 7 ('Completely True' for motivation or 'Strongly Agree' for all others).

Social interdependence was measured using three social interdependence subscales [26]: cooperation (5 items; $\alpha=0.73$ ), competition ( 5 items; $\alpha=0.82$ ), and individualism ( 5 items; $\alpha=0.85$ ).

Sociocognitive conflict regulation was measured using two scales [7]: relational conflict regulation (2 items; $r=0.55)$, and epistemic conflict regulation ( 3 items; $\alpha=0.69$ ). The lower reliability rates may be due to cultural differences, since the scales have only been used previously with French, Swiss and US samples. 
Table 1. Demographics and Descriptive Statistics

\begin{tabular}{|c|c|c|c|c|c|c|c|c|c|}
\hline \multirow[b]{2}{*}{ Variable (Maximum Value) } & \multicolumn{3}{|c|}{ Face-to-Face } & \multicolumn{3}{|c|}{ Synchronous CMC } & \multicolumn{3}{|c|}{ Asynchronous CMC } \\
\hline & Acceptance & Mild Rejection & Control & Acceptance & Mild Rejection & Control & Acceptance & Mild Rejection & Control \\
\hline \multicolumn{10}{|l|}{ Social Interdependence } \\
\hline Cooperation (35) & $28.8(2.77)$ & $21(3.22)$ & $22.6(6.58)$ & $27.71(3.5)$ & $27.71(2.98)$ & $22.33(3.39)$ & $23.71(5.94)$ & $25.17(2.64)$ & $27.71(6.65)$ \\
\hline Competition (35) & $25.6(4.62)$ & $24(6.36)$ & $22.6(6.58)$ & $20.86(3.29)$ & $21(7.9)$ & $17.83(4.12)$ & $22.71(4.92)$ & $19.5(8.6)$ & $20.14(498)$ \\
\hline Individualism (35) & $15.2(4.49)$ & $24.67(4.23)$ & $16.8(5.81)$ & $15.29(5.15)$ & $17.43(6)$ & $19.33(4.93)$ & $21.43(6.21)$ & $18(6.63)$ & $19.14(8.11)$ \\
\hline \multicolumn{10}{|l|}{ Sociocognitive Conflict Regulation } \\
\hline Relational (14) & $8.4(2.3)$ & $10(2.76)$ & $8(2.55)$ & $8.14(1.77)$ & $8.14(2.04)$ & $6.5(2.17)$ & $9.14(1.21)$ & $8.83(1.83)$ & $9.71(2.87)$ \\
\hline Epistemic (21) & $18.6(1.14)$ & $16.5(2.81)$ & $16(2.92)$ & $14.43(3.69)$ & $16.86(1.86)$ & $16(2.28)$ & $16.86(3.02)$ & $16.17(2.64)$ & $18(1.63)$ \\
\hline \multicolumn{10}{|l|}{ Motivation } \\
\hline Interest-value (98) & $83.2(8.5)$ & $66.33(6.59)$ & $78(8.63)$ & $67.71(8.65)$ & $63.29(20.26)$ & $59.17(25.81)$ & $71.71(9.84)$ & $57.67(18.61)$ & $63.43(22.16)$ \\
\hline \multicolumn{10}{|l|}{ Academic Achievement } \\
\hline Multiple Choice (5) & $4.2(1.79)$ & $4.17(1.6)$ & $3.6(1.14)$ & $3.71(1.89)$ & $4.57(0.79)$ & $4.67(0.52)$ & $4(1)$ & $3(2.19)$ & $4.57(0.79)$ \\
\hline Critical Thinking (4) & $3.5(0.14)$ & $3.43(0.03)$ & $3.62(0.18)$ & $3.23(0.26)$ & $3.42(0.19)$ & $3.48(0.03)$ & $3.71(0.07)$ & $3.63(0.16)$ & $3.58(0.21)$ \\
\hline \multicolumn{10}{|l|}{ Perceptions of Technology } \\
\hline Attitude toward Technology (28) & $25.4(2.61)$ & $21.67(5.01)$ & $18.8(5.67)$ & $21(3.42)$ & $25.14(1.95)$ & $17.5(5.24)$ & $20.57(2.51)$ & $22.33(4.08)$ & $24.86(2.48)$ \\
\hline Self-Efficacy with Technology (21) & $17.8(2.49)$ & $17(2.28)$ & $14.6(3.36)$ & $14.57(4.2)$ & $16.14(3.39)$ & $13.17(4.26)$ & $14.29(2.63)$ & $12.83(2.71)$ & $17.57(2.44)$ \\
\hline \multicolumn{10}{|l|}{ Cultural Dimensions } \\
\hline Collectivism (35) & $27(2.12)$ & $26.67(3.44)$ & $26.2(3.27)$ & $26.57(5.06)$ & $26.71(6.02)$ & $22.5(2.51)$ & $24(4.65)$ & $24.5(2.88)$ & $25.14(2.67)$ \\
\hline Uncertainty Avoidance (35) & $29.2(3.83)$ & $28.17(3.13)$ & $28.2(3.19)$ & $28.43(5.19)$ & $29.86(3.63)$ & $25.33(3.88)$ & $28.71(3.9)$ & $29.17(4.22)$ & $30(3.65)$ \\
\hline \multicolumn{10}{|l|}{ Time } \\
\hline Actual Time Spent & $4.63(1.8)$ & $4.17(1.94)$ & $4.2(1.25)$ & $6.07(6.85)$ & $5.33(3.2)$ & $4.58(2.65)$ & $4.57(1.24)$ & $4.79(4.77)$ & $4.93(2.19)$ \\
\hline Preferred Time Spent & $6.63(2.87)$ & $3.42(1.69)$ & $6.8(4.32)$ & $5(3.27)$ & $4.17(1.94)$ & $5.17(4.01)$ & $6.5(3.25)$ & $3.38(1.96)$ & $4.71(2.45)$ \\
\hline Preferred minus Actual & $2(1.41)$ & $-0.75(1.33)$ & $2.6(3.15)$ & $-1.07(4.32)$ & $-1(2.31)$ & $0.58(1.8)$ & $1.93(2.52)$ & $-1.42(4.48)$ & $-0.21(1.8)$ \\
\hline Group N & 5 & 6 & 5 & 7 & 7 & 6 & 7 & 6 & \\
\hline
\end{tabular}

Interest ( 7 items; $\alpha=0.90$ ) and value ( 7 items; $\alpha=$ 0.92) were measured using two subscales of the Intrinsic Motivation Inventory [27]. There was a strong positive correlation between interest and value $(\mathrm{r}=$ $0.76, \mathrm{p}<0.001)$, therefore the measures were aggregated to create a composite interest-value variable (14 items; $\alpha=0.94$ ).

Knowledge and understanding of whistleblowing were measured using five multiple-choice items specifically written for the articles included in the procedure. Critical thinking in the final joint essay was assessed by two raters using a rubric form. Interrater reliability was low, $\mathrm{ICC}=0.49$. Looking at descriptive statistics for the two raters, Rater 1 tended to score essays lower on average, and had a slightly larger variation compared to Rater 2 . The two sets of ratings are linearly related.

Time was measured using two questions from Saltarelli and Roseth's [3] study. Students were asked:

"How much time did you spend on the constructive controversy project, and how much time would you have preferred to spend on the project."

In our study, all participants had seven days to complete the constructive controversy procedure. Attitude toward technology ( 4 items, $\alpha_{\text {pre }}=0.90 ; \alpha_{\text {post }}=$ $0.84)$ and self-efficacy with technology ( 3 items, $\alpha_{\text {pre }}=$ $0.65 ; \alpha_{\text {post }}=0.63$ ) were measured using two subscales from Unified Theory of Acceptance and Use of Technology [28]. Both scales were measured in the preprocedure survey and in the post-procedure survey to determine if there were any changes during the constructive controversy project or if the preconceived attitudes of participants influenced the results.

\section{Analysis of Findings}

One participant switched synchronicity placement, from the randomly assigned FTF to asynchronous $\mathrm{CMC}$, due to being out of the country during the experiment. Three students dropped out after randomization, which resulted in reorganizing several constructive controversy group memberships and pro/con stances. However, the random assignments of synchronicity and belongingness were not impacted. Two participants did not fill out the pre-procedure survey and 5 participants skipped at least 1 item on the pre-procedure survey. These participants were used in the final analysis; however, they were not included in any of the pre and post comparison analyses. Two participants did not have a final essay to be scored and were not included in the analyses for critical thinking. One participant was removed from the final analysis due to failure to complete the majority of the items on the post-procedure survey. In the final sample, there were 56 participants and $66 \%$ of them were male ( 3 missing responses), with a mean age of 21.08 years (SD $=1.71 ;$ range $=18-27$ ). Table 1 provides participant descriptive statistics by boundary condition and belongingness.

\subsection{Manipulation check}

In the post-procedure survey, students were asked whether they were their partner's first choice, to examine whether their emotions varied according to the belongingness manipulation exercise. A Fisher's exact test of independence showed a statistically significant relationship between the belongingness manipulation and this question, $\chi^{2}(4, \mathrm{~N}=56)=18.22, \mathrm{p}<0.001$. 
Participants in the mild rejection condition were more likely to respond "no" and those in the control condition were more likely to respond, "I don't know".
Unexpectedly, students in the acceptance condition were least likely to say "yes". Therefore, manipulation

Table 2. Correlations Among Variables

\begin{tabular}{|c|c|c|c|c|c|c|c|c|c|c|c|c|c|}
\hline Variable & 2 & 3 & 4 & 5 & 6 & 7 & 8 & 9 & 10 & 11 & 12 & 13 & 14 \\
\hline \multicolumn{14}{|l|}{ Social Interdependence } \\
\hline 1. Cooperation & 0.07 & $-0.67^{* * *}$ & -0.12 & 0.08 & $0.36^{* *}$ & 0.09 & -0.16 & $0.28^{*}$ & 0.12 & $.28^{*}$ & 0.24 & 0.03 & -0.06 \\
\hline 2. Competition & & 0.04 & 0.22 & $0.36^{* * *}$ & $0.44^{* *}$ & 0.13 & -0.08 & 0.08 & 0.21 & 0.07 & $0.34^{* * *}$ & 0.05 & 0.21 \\
\hline 3. Individualism & & & 0.15 & 0.04 & $-0.33^{*}$ & 0.03 & 0.14 & -0.11 & 0.09 & -0.13 & 0.08 & 0.05 & 0.07 \\
\hline \multicolumn{14}{|l|}{ Sociocognitive conflict regulation } \\
\hline 4. Relational & & & & 0.12 & 0.06 & 0.13 & 0.16 & 0.15 & 0.18 & -0.03 & 0.10 & -0.05 & -0.13 \\
\hline 5. Epistemic & & & & & 0.22 & 0.21 & 0.21 & 0.13 & 0.17 & 0.11 & $0.49 * *$ & -0.15 & -0.10 \\
\hline \multicolumn{14}{|l|}{ Motivation } \\
\hline 6. Interest-value & & & & & & 0.23 & 0.00 & 0.07 & 0.23 & $.3^{*}$ & 0.18 & 0.20 & $0.32^{*}$ \\
\hline \multicolumn{14}{|l|}{ Academic achievement } \\
\hline 7. Multiple choice & & & & & & & -0.19 & 0.18 & 0.21 & 0.16 & 0.07 & $.283^{*}$ & 0.11 \\
\hline \multicolumn{14}{|l|}{ Critical Thinking } \\
\hline 8. Average score & & & & & & & & -0.16 & $-0.27 *$ & -0.22 & -0.04 & -0.07 & 0.12 \\
\hline \multicolumn{14}{|l|}{ Perceptions of technology } \\
\hline 9. Attitude towards technology & & & & & & & & & $0.63^{* *}$ & $0.35^{* *}$ & $0.28^{*}$ & 0.04 & 0.00 \\
\hline 10. Self-efficacy with technology & & & & & & & & & & $0.33^{*}$ & 0.19 & 0.04 & 0.08 \\
\hline \multicolumn{14}{|l|}{ Cultural Variables } \\
\hline 11. Collectivism & & & & & & & & & & & 0.18 & 0.11 & 0.07 \\
\hline 12. Uncertainty Avoidance & & & & & & & & & & & & -0.17 & -0.09 \\
\hline \multicolumn{14}{|l|}{ Time } \\
\hline 13. Actual Time Spent & & & & & & & & & & & & & $0.54^{* * *}$ \\
\hline 14. Preferred Time & & & & & & & & & & & & & \\
\hline
\end{tabular}

was successful for mild rejection and control, but unsuccessful for acceptance. As a result, interpretations for the different belongingness groups should be done with caution.

\subsection{Factorial analysis}

Main effects, interaction effects, and covariate effects were analyzed using a 3 (synchronicity) x 3 (belongingness) MANCOVA, using $\mathrm{CO}$ and UAI as covariates. Post hoc tests were conducted when necessary, using Bonferroni multiple comparisons. A $3 \times 3$ ANCOVA, with CO and UAI as covariates was conducted when appropriate. The necessary assumptions were met for the MANCOVAs. Homogeneity of variance was violated for the three ANCOVAs. While violation of this assumption with nearly equal group sizes is of minimal concern, these results should be interpreted with some skepticism. To conserve space, only significant results are detailed. Table 2 shows correlations between continuous variables.

5.3.1. Cultural variables - $\mathrm{CO}$ was correlated with cooperation $(r=0.28, p<0.05)$, interest-value motivation $(\mathrm{r}=0.3, \mathrm{p}<0.05)$, attitude towards technology (post) $(r=0.35, p<0.01)$, and self-efficacy with technology (post) $(r=0.33, p<0.05)$. UAI was correlated with competition $(r=0.34, p<0.01)$, epistemic conflict regulation $(\mathrm{r}=0.49, \mathrm{p}<0.01)$, and attitude towards technology (post) $(\mathrm{r}=0.28, \mathrm{p}<0.05)$.

For each ANCOVA and MANCOVA conducted, one of the assumptions is that there is no group by covariate interaction. This homogeneity of regression slopes suggests that the linear relationship between the outcome variable and cultural dimension is consistent across belongingness groups and synchronicity. This assumption was met for each ANCOVA or MANCOVA performed; thus, the cultural dimension effects are consistent across synchronicity and belongingness groups.

5.3.2. Social interdependence - Cooperation was correlated with individualism $(\mathrm{r}=-0.67, \mathrm{p}<0.01)$. MANCOVA results showed a statistically significant multivariate omnibus for UAI (Wilk's $\lambda=0.76$ ), $\mathrm{F}(3,47)=4.94, \mathrm{p}<0.01$, partial $\eta^{2}=0.24$ and for $\mathrm{CO}$ (Wilk's $\lambda=0.80$ ), $F(3,47)=3.87, p<0.02$, partial $\eta^{2}=$ 0.2. The between-subjects tests for UAI were statistically significant for competition, $\mathrm{F}(1,49)=8.34$, 
$\mathrm{p}<0.01$, partial $\eta^{2}=0.15$. The parameter estimate for UAI and competition is $\beta=0.37, \mathrm{p}<0.01$, showing a positive relationship between the two variables, controlling for $\mathrm{CO}$ and across synchronicity and belongingness groups. The between-subjects tests for $\mathrm{CO}$ were statistically significant for cooperation, $\mathrm{F}(1$, $49)=8.9, p<0.01$, partial $\eta^{2}=0.15$. The parameter estimate for $\mathrm{CO}$ and cooperation is $\beta=0.39, \mathrm{p}<0.01$, showing a positive relationship between the two variables, controlling for UAI and across synchronicity and belongingness groups. The between-subjects tests for synchronicity were statistically significant for competition, $\mathrm{F}(2,49)=3.43, \mathrm{p}=0.04$, partial $\eta^{2}=0.12$. The parameter estimates for competition and FTF is $\beta=$ $0.71, p=0.03$, suggesting that when controlling for both cultural dimensions and across belongingness groups, FTF increases competition more than SCMC.

5.3.3. Sociocognitive conflict regulation - Epistemic conflict regulation correlated with competition $(\mathrm{r}=$ $0.36, \mathrm{p}<0.01)$. MANCOVA results showed a statistically significant multivariate omnibus for UAI (Wilk's $\lambda=0.76), \mathrm{F}(2,48)=7.4, \mathrm{p}<0.01$, partial $\eta^{2}=$ 0.24 . The between-subjects test for UAI was statistically significant for epistemic conflict regulation, $\mathrm{F}(1,49)=15.1, \mathrm{p}<0.001$, partial $\eta^{2}=0.24$. The parameter estimates for UAI and epistemic conflict regulation is $\beta=0.49, \mathrm{p}<0.001$, showing a positive relationship between the two variables, controlling for $\mathrm{CO}$ and across synchronicity and belongingness groups.

5.3.4. Motivation - Interest-value correlated with cooperation $(r=0.36, p<0.01)$, competition $(r=0.44$, $\mathrm{p}<0.01)$, and individualism $(\mathrm{r}=-0.33, \mathrm{p}<0.05)$.

5.3.5. Academic achievement - ANCOVA results for critical thinking showed a statistically significant between-subjects effect for synchronicity, $\mathrm{F}(2,45)=$ 8.6, $\mathrm{p}=0.001$, partial $\eta^{2}=0.28$. Post-hoc tests revealed significant mean differences between SCMC and ACMC ( $1<0.001)$, and between SCMC and FTF ( $\mathrm{p}<$ 0.05). For both mean differences, SCMC had lower critical thinking averages compared to the other boundary conditions.

5.3.6. Time spent - Two participants had unexpectedly high values for both time variables. The extreme nature of these outliers was evident when viewing the scatterplot and their presence in the sample notably impacted results and relationships. For the remainder of the analyses involving time, these outliers were removed from the sample.
Actual time and preferred time were correlated $(\mathrm{r}=$ $0.54, \mathrm{p}<0.001)$. Preferred time spent on the procedure correlated with interest-value $(\mathrm{r}=0.32, \mathrm{p}<0.05)$. Actual time spent on the procedure correlated with multiple choice $(r=0.28, p<0.05)$.

Due to several MANCOVA assumption violations and a smaller sample size, a MANCOVA did not seem statistically appropriate for the time variables.

However, when simply comparing the group means, SCMC spent more time on average (5.4 hrs), ACMC spent the second longest (average $4.8 \mathrm{hrs}$ ), and FTF spent the least amount of time on the constructive controversy (average $4.3 \mathrm{hrs}$ ). However, the preferred time spent on the project is reversed, with FTF preferring to have spent the most $(5.4 \mathrm{hrs})$, ACMC preferring to spend the second most (average $4.9 \mathrm{hrs}$ ), and SCMC preferring to have spent the least (average $4.8 \mathrm{hrs})$.

Saltarelli found that ACMC spent more time on the constructive controversy than FTF and SCMC, but only SCMC would have preferred to spend more time on it. Our results are different, with SCMC spending more time than ACMC and FTF, with FTF preferring to spend more time on it. The mild rejection groups across synchronicity would have preferred to spend less time, while the acceptance groups for FTF and ACMC would have preferred to spend more time. For the control group, FTF and SCMC would have spent more time and the ACMC was slightly less.

5.3.7. Perceptions of technology - Attitude towards technology (pre) and self-efficacy with technology (pre) were correlated $(\mathrm{r}=0.56, \mathrm{p}<0.01)$. Attitude towards technology (post) correlated with cooperation $(\mathrm{r}=0.28, \mathrm{p}<0.05)$, attitude towards technology (pre) $(\mathrm{r}$ $=0.62, \mathrm{p}<0.01)$, and self-efficacy with technology (pre) $(r=0.39, \mathrm{p}<0.01)$. Self-efficacy with technology (post) correlated with the critical thinking average score $(\mathrm{r}=-0.27, \mathrm{p}<0.05)$. Self-efficacy with technology (post) correlated with attitude towards technology (pre) $(\mathrm{r}=0.38, \mathrm{p}<0.01)$, self-efficacy with technology (pre) $(\mathrm{r}=0.52, \mathrm{p}<0.01)$, and attitude towards technology (post) $(r=0.63, p<0.01)$.

5.3.8. Differences between pre and post-test - Initial paired samples t-tests comparing pre and post survey results were not significant for attitude towards technology and self-efficacy with technology, suggesting that the pre-procedure perceptions of technology were statistically equal to the postprocedure perceptions of technology (there is a smaller sample here due to some participants failure to complete the pre-procedure survey). After creating a 
difference variable (post minus pre), an ANOVA was conducted to determine if there were statistically significant changes in perceptions of technology between the synchronicity groups. This analysis was also not statistically significant, suggesting that the synchronicity condition did not result in any changes in perception of technology.

The difference between pre and post procedure values for self-efficacy with technology was moderately positively correlated with interest-value motivation $(r=.38, p<0.01)$, suggesting that those who were more motivated regarding the constructive controversy tended to increase in their self-efficacy with technology during the procedure, while those who were less motivated regarding the constructive controversy tended to remain about the same or decrease in their self-efficacy with technology throughout the procedure.

\subsubsection{Belongingness and perceptions of technology -} MANCOVA results showed a statistically significant multivariate omnibus for the interaction effect between belongingness and synchronicity (Wilk's $\lambda=0.66), F(8$, $88)=2.51, p<0.02$, partial $\eta^{2}=0.19$. The betweensubjects tests for belongingness and synchronicity were statistically significant for self-efficacy with technology (post), $\mathrm{F}(4,45)=2.57, \mathrm{p}=0.05$, partial $\eta^{2}=$ 0.19 , and for attitude towards technology (post), F(4, $45)=4.1, \mathrm{p}<0.01$, partial $\eta^{2}=0.27$.

The parameter estimate for self-efficacy with technology and $\mathrm{ACMC}^{*}$ mild rejection is $\beta=-1.831, \mathrm{p}$ $=0.02$, suggesting that when controlling for both cultural dimensions, in ACMC, mild rejection resulted in a lower self-efficacy with technology adjusted mean compared to the control group. The parameter estimate for attitude towards technology and ACMC*mild rejection is $\beta=-1.86, p=0.01$, suggesting that when controlling for both cultural dimensions, in ACMC, mild rejection resulted in a lower attitude towards technology adjusted mean compared to the control group.

5.3.10. Culture and perceptions of technology - The between-subjects tests for $\mathrm{CO}$ were also statistically significant for attitude towards technology (post), $\mathrm{F}(1$, $45)=4.74, p<0.04$, partial $\eta^{2}=0.1$. The parameter estimate for $\mathrm{CO}$ and attitude towards technology (post) is $\beta=0.26, \mathrm{p}<0.04$, showing a positive relationship between the two variables, controlling for UAI and across synchronicity and belongingness groups.

\section{Discussion}

The effect of boundary conditions - Constructive controversy stimulates different psychological processes and academic achievement under various boundary conditions as evidenced by the larger competition mean for FTF and decreased critical thinking average for SCMC. Similar to Saltarelli and Roseth [3], we found a statistically significant synchronicity main effect for social interdependence, specifically for competition, and a main effect for critical thinking. They found that SCMC had a larger use-of-evidence mean than FTF and FTF had a larger integrative statement mean than ACMC. We found ACMC and FTF had a larger overall critical thinking mean than SCMC. ANCOVA results showed a statistically significant relationship between critical thinking and synchronicity, with a large partial effect size of 0.28 . Prior research examined correlative relationships between competition and academic success [29].

The effects of belongingness - Mild rejection decreases attitude and self-efficacy towards the use of technology. We found interaction between synchronicity and belongingness for self-efficacy with technology and attitude towards technology. For both dependent variables, the control group in ACMC had a larger mean than the mild rejection group in ACMC. The MANCOVA for perceptions of technology resulted in a statistically significant interaction effect for belongingness and synchronicity. When controlling for both cultural dimensions in the ACMC boundary condition, mild rejection resulted in lower self-efficacy with technology and lower attitude towards technology compared to the control group with large partial effect sizes of 0.19 and 0.27 , respectively. These findings build on prior research conducted by Saltarelli and Roseth [3] who found a statistically significant belongingness main effect for self-efficacy with technology, where acceptance resulted in a larger selfefficacy with technology mean than the control condition. Unexpectedly, results did not support our hypotheses regarding the effects of mild rejection across all boundary conditions nor was there evidence for the additive effects of satisfying student belongingness needs.

The effects of culture - Our findings showed that collectivism increased attitude towards technology (medium effect size of 0.1 ) and cooperation (large partial effect size of 0.15 ), and uncertainty avoidance increased competition (large partial effect size of 0.15 ) and epistemic behavior (large partial effect size 0.24). 
These results support our hypothesis that cultural dimensions affect constructive controversy outcomes and generalize across boundary conditions.

Unlike our predictions, our sample of students who scored high on $\mathrm{CO}$ and UAI did not suffer the deleterious effects of ACMC. Instead, culture moderated the relationship between students' problem solving and collaboration skills and motivation. $\mathrm{CO}$ was positively correlated with cooperation, interestvalue motivation, and attitude towards and self-efficacy towards technology. As such, higher levels of $\mathrm{CO}$ in our Arabic and Asian sample promoted positive psychological processes and motivation. The UAI cultural variable was positively correlated with competition, epistemic conflict regulation, and attitude towards technology. In other words, higher UAI in our sample promoted competition but surprisingly with an epistemic approach to conflict, while keeping a positive attitude towards technology.

\section{Limitations}

Our results were limited by the characteristics of the sample and by the operationalizations of the dependent variables. Our ability to find statistically significant relationships between the constructs of interest is dependent on the validity and reliability of our chosen measures, most of which are Western-based scales.

Challenges with technology due to the UAE government restrictions on digital networks could have affected our sample differently than those in the original US study conducted by Saltarelli and Roseth [3].

\section{Conclusion}

The results of this study have implications for both theory and practice. First, the difference in critical thinking in the SCMC boundary condition is cause for concern for online learning. One argument in favor of synchronous online learning revolves around an attempt to create a classroom-like environment to stimulate learning. However, at least in the present study, students in the SCMC boundary demonstrated the lowest levels of critical thinking. It could be that students in the SCMC condition in our study perceived the videoconferencing system as an extension of social media, rather than a learning tool, resulting in low scores on critical thinking. If this is a robust effect, and critical thinking is an outcome of interest in education,
SCMC should be modified to incorporate more structure to avoid using the medium as a social activity and focus the time allocated to complete the learning objectives.

The differences in the effect of boundary conditions between the current study and Saltarelli's study [3] require explanation. Either one of our studies is flawed or, more likely, moderating variables not included in the studies explain the different results. Three important differences in the samples for the two studies exist which could explain the results, at least in part. For Saltarelli and Roseth [3], competition was higher in ACMC whereas it was higher in FTF in the present study. While Saltarelli and Roseth's [3] sample was majority female, the current sample was majority male. The greater presence of social cues in the FTF environment could stimulate competition in male participants and suppress competition in female participants. It should be noted, that the FTF activity used up the least amount of time to complete the learning outcomes.

Culture represents a second difference between the two samples. Saltarelli and Roseth's sample [3] was comprised primarily of US citizens while the present sample was comprised primarily of students from Asia and the Middle East. Asian and Arab cultures tend to be higher in collectivism and moderately lower uncertainty avoidance than the US. As expected, collectivism increased attitudes towards technology and cooperation. Interestingly UAI increased competition but with an epistemic approach to conflict resolution across boundary conditions and belongingness groups. Overall, the role of culture had a positive moderating effect in regulating motivation and behaviors in constructive controversy and certainly deserves additional study. It is worth highlighting that students in our current sample do not have experience in online courses (as the university does not offer distance education) compared to the US sample. In practice, the design of courses in cultures high in uncertainty avoidance should provide maximum access to information that enables students to create structure and understanding, thereby reducing uncertainty. Although more research is needed to understand the effects of these variables, the design of online courses should take gender, culture, and online experience into account.

The effect of mild rejection is interesting. In the FTF and SCMC environments, mild rejection produced no identifiable effects. However, in the ACMC 
condition, students who experienced rejection reported more negative attitudes toward technology and reduced technology-related efficacy. Since the ACMC environment is entirely technology dependent, these adverse reactions to mild rejection could partially explain the high dropout rate commonly reported in online learning. Specifically, poor attitude toward, and reduced efficacy with, technology could mediate the relationship between perceived rejection and withdrawal from class. It is left to future research to confirm this theory. The fact that mild rejection did not have lasting effects on attitudes in the FTF and SCMC environments is easily explained. The social cues that occur when students interact in those two environments overwhelm the effect of artificially induced mild rejection and eliminate its effects.

For online, asynchronous learning, instructional designers need to identify mechanisms that prevent students from experiencing rejection and include activities that help address mild rejection. Such activities could embed interactions and scenarios between students that allow them to learn about team building, negotiation, and collaborative skills.

Our study demonstrates that constructive controversy can be used effectively in a well-designed and structured online learning. We also provide support for Saltarelli and Roseth's [3] claim that boundary conditions and mild rejection matter, though the direction of the differences was not always the same across the two studies. Finally, our study demonstrates that culture is an important determinant of student experiences in online learning and hints at the importance of gender and previous experience with non-traditional education in the design and conduct of computer-mediated instruction.

\section{References}

[1] Romano, J., Wallace, T.L., Helmick, I.J., Carey, L.M., and Adkins, L. (2005). Study Procrastination, Achievement, And Academic Motivation in Web-Based and Blended Distance Learning. The Internet and Higher Education, 8(4), 299-305.

[2] Johnson, D.W., Johnson, R.T., and Tjosvold, D. (2012). Constructive controversy: The Value of Intellectual Opposition. The Handbook of Conflict Resolution: Theory and Practice.

[3] Saltarelli, A.J., and Roseth, C.J. (2014). Effects of Synchronicity and Belongingness on Face-To-Face and
Computer-Mediated Constructive Controversy. Journal of Educational Psychology, 106(4), 946-960.

[4] Johnson, D.W., and Johnson, R.T. (1989). Cooperation and Competition: Theory and Research. Edina, MN, US: Interaction Book Company.

[5] Johnson, D.W., and Johnson, R.T. (2009). Energizing Learning: The Instructional Power of Conflict. Educational Researcher, 38(1), 37-51.

[6] Darnon, C., Buchs, C., and Butera, F. (2002). Epistemic and Relational Conflicts in Sharing Identical Vs Complementary Information During Cooperative Learning. Swiss Journal of Psychology, 61(3), 139-151.

[7] Darnon, C., Muller, D., Schrager, S.M., Pannuzzo, N., and Butera, F. (2006). Mastery and Performance Goals Predict Epistemic and Relational Conflict Regulation. Journal of Educational Psychology, 98(4), 766-776.

[8] Brochu, M. (2017). Cyber Bullying: A Quantitative Study on The Perceptions and Experiences of Female Emirati University Students.

[9] Ryan, R.M., and Deci, E.L. (2000). Self-Determination Theory and The Facilitation of Intrinsic Motivation, Social Development, And Well-Being. American Psychologist, 55(1), 68-78.

[10] Martin, A.J., and Dowson, M. (2009). Interpersonal Relationships, Motivation, Engagement, And Achievement: Yields for Theory, Current Issues, And Educational Practice. Review of Educational Research, 79(1), 327-365.

[11] Juvonen, J., and Wentzel, K.R. (1996). Social Motivation: Understanding Children's School Adjustment. Cambridge University Press.

[12] Wallace, R.M. (2003). Online Learning in Higher Education: A Review of Research on Interactions Among Teachers and Students. Education, Communication and Information, 3(2), 241-280.

[13] Roseth, C.J., Saltarelli, A.J., and Glass, C.R. (2011). Effects of Face-To-Face and Computer-Mediated Constructive Controversy on Social Interdependence, Motivation, And Achievement. Journal of Educational Psychology, 103(4), 804-820.

[14] Al-Harthi, A.S. (2010). Learner Self-Regulation in Distance Education: A Cross-Cultural Study. The American Journal of Distance Education, 24(3), 135-150.

[15] Alsubaie, M.A. (2015). Examples of Current Issues in The Multi-Cultural Classroom. Journal of Education and Practice, 6(10), 86-89. 
[16] King, E.W. (2002). Ethnicity. In D. L. Levinson, P. W. Cookson, Jr., and A. R. Sadowski (Eds.), Education and Sociology: An Encyclopedia (pp. 247-253). New York: Routledge Falmer.

[17] Hofstede, G. (2001). Culture's Consequences: Comparing Values, Behaviors, Institutions, and Organizations Across Nations, 2nd ed. Sage, Thousand Oaks, CA.

[18] Sheldon, K.M., Elliot, A.J., Kim, Y., and Kasser, T. (2001). What Is Satisfying About Satisfying Events? Testing 10 Candidate Psychological Needs. Journal of Personality and Social Psychology, 80(2), 325-339.

[19] Khattab, A. (2015). How Arab Executives Learn. Michigan State University.

[20] Nisbett, R. (2004). The Geography of Thought: How Asians And Westerners Think Differently...and Why. Simon and Schuster.

[21] Deutsch, M., Coleman, P.T., and Marcus, E. (2011). The Handbook of Conflict Resolution: Theory and Practice. John Wiley and Sons.

[22] Sato, T. (2005). The Eysenck Personality Questionnaire Brief Version: Factor Structure and Reliability. The Journal of Psychology, 139(6), 545-552.

[23] Twenge, J. M., Baumeister, R. F., Tice, D. M., and Stucke, T. S. (2001). If You Can't Join Them, Beat Them: Effects of Social Exclusion on Aggressive Behavior. Journal of Personality and Social Psychology, 81(6), 1058-1069.

[24] Romero-Canyas, R., Downey, G., Reddy, K.S., Rodriguez, S., Cavanaugh, T.J., and Pelayo, R. (2010). Paying to Belong: When Does Rejection Trigger Ingratiation? Journal of Personality and Social Psychology, 99(5), 802823.

[25] Yoo, B., Donthu, N., and Lenartowicz, T. (2011). Measuring Hofstede's Five Dimensions of Cultural Values at The Individual Level: Development and Validation of CVSCALE. Journal of International Consumer Marketing, 23(3-4), 193-210.

[26] Johnson, D.W., and Norem-Hebeisen, A. (1977). Attitudes Toward Interdependence Among Persons and Psychological Health. Psychological Reports, 40(3), 843850 .

[27] Ryan, R.M. (1982). Control and Information in The Intrapersonal Sphere: An Extension of Cognitive Evaluation Theory. Journal of Personality and Social Psychology, 43(3), 450-461.
[28] Venkatesh, V., Morris, M., Davis, G., and Davis, F. (2003). User Acceptance of Information Technology: Toward a Unified View. MIS Quarterly, 27(3), 425-478.

[29] Wentzel, K.R. (1999). Social-Motivational Processes and Interpersonal Relationships: Implications for Understanding Motivation at School. Journal of Educational Psychology, 91(1), 76-97. 